\title{
ATOMICITY OF MAPPINGS
}

\author{
JANUSZ J. CHARATONIK \\ Mathematical Institute \\ University of Wrocław \\ Pl. Grunwaldzki 2/4, 50-384 \\ Wrocław, POLAND \\ Instituto de Matemáticas \\ Universidad Nacional Autónoma de México \\ Circuito Exterior, Ciudad Universitaria \\ 04510 México, D.F., MEXICO \\ and \\ WLODZIMIERZ J. CHARATONIK \\ Mathematical Institute \\ University of Wrocław \\ Pl. Grunwaldzki 2/4, 50-384 \\ Wrocław, POLAND \\ Departamento de Matemáticas \\ Facultad de Ciencias \\ Universidad Nacional Autónoma de México \\ Circuito Exterior, Ciudad Universitaria \\ 04510 México, D.F., MEXICO
}

(Received December 16, 1996 and in revised form December 11, 1997)

\begin{abstract}
A mapping $f: X \rightarrow Y$ between continua $X$ and $Y$ is said to be atomic at a subcontinuum $K$ of the domain $X$ provided that $f(K)$ is nondegenerate and $K=f^{-1}(f(K))$. The set of subcontinua at which a given mapping is atomic, considered as a subspace of the hyperspace of all subcontinua of $X$, is studied. The introduced concept is applied to get new characterizations of atomic and monotone mappings. Some related questions are asked.
\end{abstract}

KEY WORDS AND PHRASES: Atomic, composition factor property, continuum, contratomic, hyperspace, mapping, monotone.

1991 AMS SUBJECT CLASSIFICATION CODES: Primary 54E40, 54F15. Secondary 54B15.

\section{INTRODUCTION}

All spaces considered in the paper are assumed to be metric, and a mapping means a continuous function. A continuum means a compact connected space. Recall that a mapping $f: X \rightarrow Y$ betwee.1 continua $X$ and $Y$ is said to be monotone if the inverse image of each point of $Y$ (equivalently, of each subcontinuum of $Y$ ) is connected. A surjective mapping $f: X \rightarrow Y$ between continua $X$ and $Y$ is said to be atomic provided that, for each subcontinuum $K$ of $X$ such that $f(K)$ is nondegenerate, $K=f^{-1}(f(K))$. The notion of an atomic mapping was introduced by $\mathrm{R}$. D. Anderson in [1] to describe special decompositions of continua. Soon, atomic mappings turned out to be important tools in continuum theory and proved to be interesting by themselves, and several of their properties have been discovered, e.g. in [3], [5] and [6]. The following fact on atomic mappings is known (see [3, Theorem 1, p. 49] and [6, (4.14), p. 17]).

Fact. Every atomic mapping of a continuum is (hereditarily) monotone.

The paper consists of two parts. In the first one the composition factor property is discussed for the class of atomic mappings. The second part deals with the family of subcontinua of the domain continuum $X$ at which a given mapping $f: X \rightarrow Y$ is atomic. In particular, atomic mappings as well as monotone ones are characterized by conditions concerning the structure of this family. The paper is supplied with a number of examples; open problems posed in both parts of the paper indicate some directions of a further study in the area 
The following standard notation will be used. $\mathbb{N}, \mathbb{R}$ and $\mathbb{C}$ stand for the sets of positive integers, reals, and complex numbers, respectively, equipped with their natural topologies, if needed. In the plane $\mathbb{R}^{2}$ the symbol $(x, y)$ means a point having $x$ and $y$ as its Cartesian coordinates.

\section{COMPOSITION FACTOR PROPERTY}

We say that a class $\mathcal{M}$ of mappings has the composition factor property if the composition $g \circ h$ of mappings $h$ and $g$ is in $\mathcal{M}$ only if $g \in \mathcal{M}$.

T. Maćkowiak asked in [6, (5.22), p. 33] if the class of atomic mappings has the composition factor property, and conjectured that it does. Later, in [7, Chapter 1, Example, p. 7] he has answered his question in the negative. Another answer was given by E. E. Grace and E. J. Vought in [4, Section 4, p 140], who have shown that for the natural projection $f$ of the circle of pseudo-arcs $X$ onto the circle $Z$ (which is clearly an atomic mapping) there exist a continuum $Y$ and two mappings $h: X \rightarrow Y$ and $g: Y \rightarrow Z$, such that $f$ can be factored as the composition $g \circ h$ and $g$ is not atomic. Both the conjecture of Maćkowiak and its negative solution by himself and by Grace and Vought show that the composition factor property for the class of the atomic mappings should be studied in a more detailed way, and that there are interesting problems around this property worthwhile clarifying.

In general, the following problem can be posed.

Problem 1.1. Let $X, Y$ and $Z$ be continua, and let $h: X \rightarrow Y$ and $g: Y \rightarrow Z$ be surjective mappings. Determine conditions concerning (a) the continuum $X$, (b) the continuum $Y$, (c) the mapping $h$, under which the implication holds

$$
\text { if } g \circ h \text { is atomic, then } g \text { is atomic }
$$

To be more precise, introduce the following definition.

Definition 1.3. A class of $\mathcal{C}$ of continua is said to have the composition factor property for a class $\mathcal{M}$ of mappings provided that for each continuum $X \in \mathcal{C}$ if the composition $g \circ h$ defined on $X$ is in $\mathcal{M}$, then $g$ is in $\mathcal{M}$.

A continuum is said to be decomposable if it is the union of two its proper subcontinua. Otherwise it is said to be indecomposable. A continuum is said to be hereditarily decomposable (hereditarily indecomposable) provided that each of its nondegnerate subcontinua is decomposable (indecomposable, respectively). Finally recall that a space $X$ is said to be homogeneous provided that for every two points $p$ and $q$ of $X$ there is a homeomorphism $f: X \rightarrow X$ such that $f(p)=q$.

It is shown in [2] that the circle of pseudo-arcs (that has been used in [4] as mentioned above) is constructed in the Euclidean plane, is decomposable, and is homogeneous. Therefore the result of Grace and Vought can be formulated even in a stronger form, as follows.

Theorem 1.4. The following classes of continua do not have the composition factor property for the class of atomic mappings: plane continua, decomposable continua, homogeneous continua, as well as the intersection of any of these classes.

On the other hand, it is known that each atomic mapping defined on an arcwise connected continuum is a homeomorphism provided that the image continuum is nondegenerate (see $[6,(6.3)$, p. 51]). Since the class of homeomorphisms obviously has the composition factor property $[6,(5.14), \mathrm{p} .32]$, the following result is immediate.

Statement 1.5. The class of arcwise connected continua (of locally connected ones, in particular) has the composition factor property for the class of atomic mappings.

Thus the following problem is natural.

Problem 1.6. Determine the classes of continua which have the composition factor property for the class of atomic mappings.

Two particular questions related to this problem are of a special interest. 
Question 1.7. Does the class of hereditarily decomposable continua have the composition factor property for the class of atomic mappings?

Question 1.8. Does the class of hereditarily indecomposable continua have the composition factor property for the class of atomic mappings?

A surjective mapping $h: X \rightarrow Y$ between continua $X$ and $Y$ is said to be weakly confluent provided that for each subcontinuum $Q$ of $Y$ there is a subcontinuum $C$ of $X$ such that $h(C)=Q$. In connection with Problem 1.1, part (c), recall the following result (see [6, (5.29), p. 35]).

Proposition 1.9. If the mapping $h: X \rightarrow Y$ is weakly confluent, then implication (1.2) is satisfied.

Note that the converse to Proposition 1.9 is not true. Namely we have the following example

Example 1.10. There are mappings $h: X \rightarrow Y$ and $g: Y \rightarrow Z$ such that the composition $g \circ h$ and the second mapping $g$ are atomic, while $h$ is not weakly confluent.

Proof. Take as $X$ the well known $\sin (1 / x)$-curve $S$ defined by

$$
S=\left\{(0, y) \in \mathbb{R}^{2}: y \in[-1,1]\right\} \cup\left\{(x, \sin (1 / x)) \in \mathbb{R}^{2}: x \in(0,1]\right\},
$$

and let $L$ be the limit segment of $S$. Identify the two end points of $L$ and denote by $h: X \rightarrow Y$ the identification mapping. Thus $Y$ is the union of a half line and the circle $h(L)$. Now let us shrink $h(L)$ to a point, and let $g: Y \rightarrow Z$ be the quotient mapping. Thus $Z$ is an arc, both $g$ and $g \circ h$ are atomic, while $h$ is not weakly confluent.

A surjective mapping $h: X \rightarrow Y$ between continua $X$ and $Y$ is said to be confluent provided that for each subcontinuum $Q$ of $Y$ and for every component $C$ of the inverse image $h^{-1}(Q)$ we have $h(C)=Q$. Since a continuum $Y$ is hereditarily indecomposable if and only if each mapping from a continuum onto $Y$ is confluent (compare [6, (6.11), p. 53]), and since each confluent mapping obviously is weakly confluent, we get a corollary to Proposition 1.9, which is related to part (b) of Problem 1.1.

Corollary 1.12. If the continuum $Y$ is hereditarily indecomposable, then implication (1.2) is satisfied.

\section{ATOMICITY}

Given a continuum $X$ with a metric $d$, we let $2^{X}$ denote the hyperspace of all nonempty closed subsets of $X$ equipped with the Hausdorff metric $H$ defined by

$$
H(A, B)=\max \{\sup \{d(a, B): a \in A\}, \sup \{d(b, A): b \in B\}\}
$$

(equivalently: with the Vietoris topology, see e.g. $[8,(0.1)$, p. 1 and $(0.12)$, p. 10]. Further, we denote by $C(X)$ the hyperspace of all subcontinua of $X$, i.e., of all connected elements of $2^{X}$, and by $F_{1}(X)$ the hyperspace of singletons. The reader is referred to Nadler's book [8] for needed information on the structure of hyperspaces. In particular, the following is well known (see [8, Theorem (1.13), p. 65]).

Fact 2.1. For each continuum $X$ the hyperspace $C(X)$ is a subcontinuum of the hyperspace $2^{X}$.

Given a mapping $f: X \rightarrow Y$ between continua $X$ and $Y$, we consider mappings (called the induced ones)

$$
2^{f}: 2^{X} \rightarrow 2^{Y} \text { and } C(f): C(X) \rightarrow C(Y)
$$

defined by

$$
2^{f}(A)=f(A) \text { for every } A \in 2^{X} \quad \text { and } \quad C(f)(A)=f(A) \text { for every } A \in C(X) .
$$

Thus, by Fact 2.1, the following is obvious.

Fact 2.2. For every continua $X$ and $Y$ and for each mapping $f: X \rightarrow Y$ we have $2^{f} \mid C(X)=C(f)$ A proof of the next fact is straightfoward.

Fact 2.3. Let a mapping $f: X \rightarrow Y$ between continua $X$ and $Y$ be given Then $C(f)\left(F_{1}(X)\right) \subset F_{1}(Y)$. 
For an arbitrary surjection $f: X \rightarrow Y$ between continua we consider subcontinua of $X$ at which the mapping satisfies the atomicity condition. More precisely, given a surjective mapping $f: X \rightarrow Y$ between continua $X$ and $Y$, we denote by $\mathcal{A}(X, f)$ the family of all subcontinua $K$ of $X$ such that $f(K)$ is nondegenerate and the equality $K=f^{-1}(f(K))$ holds, i.e.,

$$
\mathcal{A}(X, f)=\left\{K \in C(X) \backslash(C(f))^{-1}\left(F_{1}(Y)\right): K=f^{-1}(f(K))\right\} .
$$

Thus the following result is a consequence of this definition.

Statement 2.5. Let a mapping $f: X \rightarrow Y$ between continua $X$ and $Y$ be given. Then

$$
\begin{gathered}
X \in \mathcal{A}(X, f), \text { so } \mathcal{A}(X, f) \text { is nonempty; } \\
\mathcal{A}(X, f) \subset C(X) \backslash(C(f))^{-1}\left(F_{1}(Y)\right) .
\end{gathered}
$$

Further, we put

$$
\mathcal{B}(X, f)=\mathcal{A}(X, f) \cup(C(f))^{-1}\left(F_{1}(Y)\right) .
$$

Proposition 2.9. For every decreasing sequence of continua belonging to $\mathcal{B}(X, f)$ the limit of the sequence also is in $\mathcal{B}(X, f)$.

Proof. For each $n \in \mathbb{N}$ assume $K_{n} \in \mathcal{B}(X, f)$ and $K_{n+1} \subset K_{n}$. Put $K=\operatorname{Lim} K_{n}$ and note that $K=\bigcap\left\{K_{n}: n \in \mathbb{N}\right\}$.

Consider two cases. First, if $K_{n} \in(C(f))^{-1}\left(F_{1}(Y)\right)$ for almost all $n \in \mathbb{N}$, then $K \in(C(f))^{-1}\left(F_{1}(Y)\right)$, too, because $F_{1}(Y)$ is compact, and so is its preimage under $C(f)$. Thus $K \in \mathcal{B}(X, f)$. Second, if $K_{n} \in \mathcal{A}(X, f)$ for almost all $n \in \mathbb{N}$, then $f^{-1}\left(f\left(K_{n}\right)\right)=K_{n}$ for these indices $n$, and we have

$$
\begin{array}{r}
f^{-1}(f(K))=f^{-1}\left(f\left(\operatorname{Lim} K_{n}\right)\right)=f^{-1}\left(\operatorname{Lim} f\left(K_{n}\right)\right)=f^{-1}\left(\bigcap\left\{f\left(K_{n}\right): n \in \mathbb{N}\right\}\right)= \\
\bigcap\left\{f^{-1}\left(f\left(K_{n}\right)\right): n \in \mathbb{N}\right\}=\bigcap\left\{K_{n}: n \in \mathbb{N}\right\}=K .
\end{array}
$$

Thus either $K \in \mathcal{A}(X, f)$ (if $f(K)$ is not a singleton), or $K \in(C(f))^{-1}\left(F_{1}(Y)\right.$ ) (if $f(K)$ is degenerate). Consequently, $K \in \mathcal{B}(X, f)$ by (2.8). The proof is then complete.

The example below shows that the conclusion of Proposition 2.9 is not true for arbitrary sequences of continua In particular, the assumption "decreasing" cannot be replaced by "increasing" in Proposition 2.9.

Example 2.10. There is a continuum $X$, an increasing sequence of subcontinua $K_{n}$ in $X$ and a monotone mapping $f: X \rightarrow Y$ such that $K_{n} \in \mathcal{B}(X, f)$ for each $n \in \mathbb{N}$, while $\operatorname{Lim} K_{n} \notin \mathcal{B}(X, f)$

Proof. Let $S$ be the $\sin (1 / x)$-curve defined by (1.11). Put $A=\left\{(0, y) \in \mathbb{R}^{2}: y \in[1,2]\right\}$, and define $X=S \cup A$. Let $Y=[0,1]$, and let $f: X \rightarrow Y$ be the projection defined by $f(x, y)=x$. For each $n \in \mathbb{N}$ let $K_{n}=f^{-1}([1 /(n+1), 1])$. Then $K_{n} \in \mathcal{A}(X, f) \subset \mathcal{B}(X, f)$, and $\operatorname{Lim} K_{n}=S$. Since $f(S)=Y$, we have $f^{-1}(f(S))=X$, and thus $\operatorname{Lim} K_{n} \notin \mathcal{B}(X, f)$, as claimed. The argument is complete.

Theorem 2.11. For each surjective mapping $f: X \rightarrow Y$ between continua $X$ and $Y$ the following assertions are equivalent:

$$
\begin{gathered}
f \text { is atomic; } \\
\mathcal{A}(X, f)=C(X) \backslash(C(f))^{-1}\left(F_{1}(Y)\right) ; \\
\mathcal{B}(X, f)=C(X) ;
\end{gathered}
$$$$
\mathcal{A}(X, f) \text { is an open subset of } C(X) \text {. }
$$ 
Proof. Equivalence of (2.12) and (2.13) is evident from the definitions, and (2.14) is another form of (2 13) by (2.8). Thus (2.12), (2.13) and (2.14) are equivalent. The implication from (2.13) to (215) is obvious. We will show that (2.15) implies (2.14). To this aim recall that an order arc in the hyperspace $C(X)$ is a family $\mathcal{L}$ of subcontinua of $X$ such that for every two members $A$ and $B$ of $\mathcal{L}$ we have either $A \subset B$ or $B \subset A$. Let $p$ be an arbitrary point of $X$ and let $\mathcal{L}$ denote an order arc in $C(X)$ from $\{p\}$ to $X$. By (2.15) the intersection $\mathcal{L} \cap \mathcal{A}(X, f)$ is an open subset of $\mathcal{L}$. Denote by $\mathcal{C}$ the component of the intersection that contains $X$, and by $K$ the (only) element of the boundary of $\mathcal{C}$ in $\mathcal{L}$. Then $K \notin \mathcal{L} \cap \mathcal{A}(X, f)$. Take a decreasing sequence $\left\{K_{n} \in \mathcal{C}: n \in \mathbb{N}\right\}$ of subcontinua of $X$ converging to $K$. Then $K=\bigcap\left\{K_{n}: n \in \mathbb{N}\right\}$, and since $K_{n} \in \mathcal{C} \subset \mathcal{A}(X, f) \subset \mathcal{B}(X, f)$ by (28), we infer from Proposition 2.9 that $K \in \mathcal{B}(X, f)$. Since $K \notin \mathcal{A}(X, f)$, we have $K \in(C(f))^{-1}\left(F_{1}(Y)\right)$ by (2.8), whence it follows that $f(K) \in F_{1}(Y)$, and therefore, by the definition of an order arc, the subarc of $\mathcal{L}$ from $\{p\}$ to $K$ is contained in $(C(f))^{-1}\left(F_{1}(Y)\right)$, thus in $\mathcal{B}(X, f)$, while the rest of the order $\operatorname{arc} \mathcal{L}$, i.e., $\mathcal{C}$, is contained in $\mathcal{A}(X, f)$ by its definition. So, we conclude by (2.8) that the whole order arc $\mathcal{L}$ is contained in $\mathcal{B}(X, f)$. Now, since $p$ was chosen as an arbitrary point of $X$, we infer from $\mathcal{L} \subset \mathcal{B}(X, f)$ that $C(X) \subset \mathcal{B}(X, f)$, whence (2.14) follows. The proof is complete.

The next theorem is a characterization of monotone mappings in the introduced terms

Theorem 2.16. For each surjective mapping $f: X \rightarrow Y$ between continua $X$ and $Y$ the following assertions are equivalent:

$$
\begin{gathered}
f \text { is monotone; } \\
C(f)(\mathcal{B}(X, f))=C(Y) .
\end{gathered}
$$

Proof. Assume $f$ is monotone. Since one inclusion of equality (2.18) is obvious, we have to show the other one. Let $L$ be a nondegenerate subcontinuum of $Y$, i.e. $L \in C(Y) \backslash F_{1}(Y) \quad$ Putting $K=f^{-1}(L)$ we see that $K$ is a continuum by monotoneity of $f$, and we have $f^{-1}(f(K))=f^{-1}(L)=K$ Thus $K \in \mathcal{A}(X, f) \subset \mathcal{B}(X, f)$ by (2.4) and (2.8), whence $L \in C(f)(\mathcal{B}(X, f))$, and (2.18) follows

Assume equality (2.18) holds. Take a point $y_{0} \in Y$ and, to show that $f$ is monotone, i.e., that $f^{-1}\left(y_{0}\right)$ is connected, consider for each positive integer $n$ the component $L_{n}$ containing $y_{0}$ of a closed $1 / n$-neighborhood about $y_{0}$ in $Y$. Thus $L_{n}$ is a nondegenerate subcontinuum of $Y$, i e, $L_{n} \in C(Y) \backslash F_{1}(Y)$. Again by (2.18) we infer that $L_{n} \in C(f)(\mathcal{A}(X, f))$, whence (for each $n$ ) there exists a nondegenerate subcontinuum $K_{n}$ of $X$ such that $f\left(K_{n}\right)=L_{n}$ and $K_{n}=f^{-1}\left(f\left(K_{n}\right)\right)=f^{-1}\left(L_{n}\right)$ Observe that for each $n$ we have $L_{n+1} \subset L_{n}$, i.e., that the sequence $\left\{L_{n}\right\}$ is decreasing, and that $\left\{y_{0}\right\}=\bigcap\left\{L_{n}: n \in \mathbb{N}\right\}$. Thus it follows from the equality $K_{n}=f^{-1}\left(L_{n}\right)$ that the sequence $\left\{K_{n}\right\}$ is decreasing, too, and we have

$$
f^{-1}\left(y_{0}\right)=f^{-1}\left(\bigcap\left\{L_{n}: n \in \mathbb{N}\right\}\right)=\bigcap\left\{f^{-1}\left(L_{n}\right): n \in \mathbb{N}\right\}=\bigcap\left\{K_{n}: n \in \mathbb{N}\right\} .
$$

Thus $f^{-1}\left(y_{0}\right)$ is a continuum as the intersection of a decreasing sequence of continua $K_{n}$ The proof is then complete.

Theorems 2.11 and (indirectly) 2.16 motivate the following question.

Question 2.19. What is the Borel class of the set $\mathcal{A}(X, f)$ considered as a subspace of the hyperspace $C(X)$ ?

In connection with (2.6) of Statement 2.5 observe that the mapping $h$ of the unit circle $S^{1}=\{z \in \mathbb{C}:|z|=1\}$ (where $\mathbb{C}$ stands for the complex plane) onto itself defined by $h(z)=z^{2}$ has the property that the whole $S^{1}$ is the only element of $\mathcal{A}(X, f)$, i.e., $\mathcal{A}(X, f)=\left\{S^{1}\right\}$. Generalizing this phenomenon, consider the following class of mappings.

Definition 2.20. A surjective mapping $f: X \rightarrow Y$ between continua $X$ and $Y$ is called contratomic provided that $\mathcal{A}(X, f)=\{X\}$. In other words, a nonconstant mapping $f$ is contratomic if the only 
subcontinuum $K$ of $X$ having nondegenerate image and satisfying the equality $K=f^{-1}(f(K))$ is $X$ itself.

The above considered mapping $h$ of $S^{1}$ onto itself is an example of a contratomic mapping. Note that the class of contratomic mappings does not contain homeomorphisms (moreover, any homeomorphism, being atomic, is not contratomic). In connection with this observe that if again $h: S^{1} \rightarrow S^{1}$ is defined by $h(z)=z^{2}$ and $g: S^{1} \rightarrow S^{1}$ is the identity, then the compositions $h \circ g=h$ and $g \circ h=h$ are contratomic, while $g$ is not. This leads to the following observation.

Observation 2.21. The class of contratomic mappings does not have the composition factor property.

Proposition 2.22. Let $h: X \rightarrow Y$ and $g: Y \rightarrow Z$ be surjective mappings between continua $X, Y$ and $Z$, respectively. If either $h$ or $g$ is contratomic, then the composition $g \circ h$ is contratomic, too

Proof. For any $K \subset X$ we have

$$
K \subset h^{-1}(h(K)) \text { and } h(K) \subset g^{-1}(g(h(K)))
$$

Let $K \in C(X) \backslash\{X\}$. If $h$ is contratomic, then $K \subsetneq h^{-1}(h(K))$; and if $g$ is contratomic, then $h(K) \subsetneq g^{-1}(g(h(K)))$, which leads to

$$
K \subset h^{-1}(h(K)) \subsetneq h^{-1}\left(g^{-1}(g(h(K)))\right)
$$

since $h$ is surjective. Putting $f=g \circ h$, we get $K \subsetneq h^{-1}\left(g^{-1}(g(h(K)))\right)=f^{-1}(f(K))$ in either of the two considered cases The proof is then complete.

\section{REFERENCES}

[1] ANDERSON, R.D., Atomic decompositions of continua, Duke Math. J. 24 (1956), 507-514

[2] BING, R.H. and JONES, F.B., Another homogeneous plane continuum, Trans. Amer. Math. Soc. 90 (1959), 171-192.

[3] EMERYK, A. and HORBANOWICZ, Z., On atomic mappings, Colloq. Math. 27 (1973), 49-55

[4] GRACE, E.E. and VOUGHT, E.J., Four mapping problems of Maćkowiak, Colloq. Math. 69 (1995), 133-141.

[5] HOSOKAWA, H., Some remarks on the atomic mappings, Bull. Tokyo Gakugei Univ. (4), 40 (1988), 31-37.

[6] MAĆKOWIAK, T., Continuous mappings of continua, Dissertationes Math. (Rozprawy Mat.) 158 (1979), 1-91.

[7] MAĆKOWIAK, T., Singular arc-like continua, Dissertationes Math. (Rozprawy Mat.) 257 (1986), 1-35.

[8] NADLER, S.B., Jr., Hyperspaces of Sets, M. Dekker, 1978 


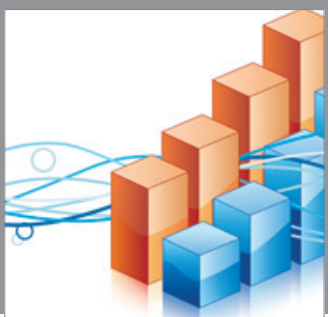

Advances in

Operations Research

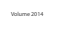

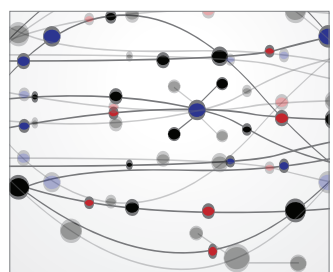

\section{The Scientific} World Journal
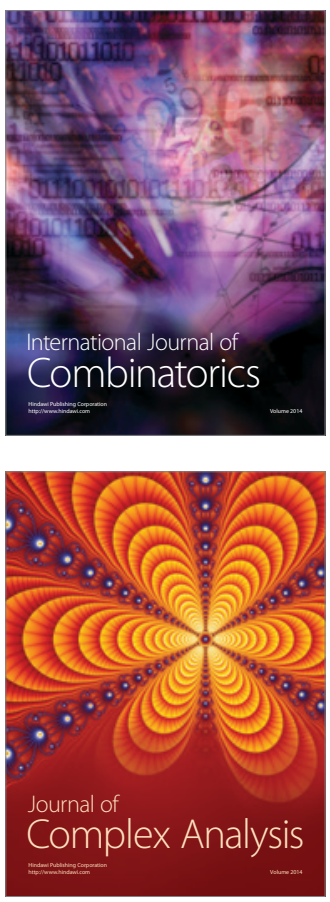

International Journal of

Mathematics and

Mathematical

Sciences
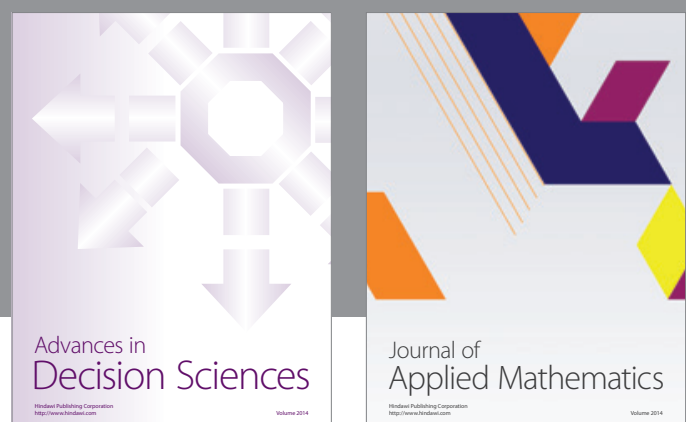

Journal of

Applied Mathematics
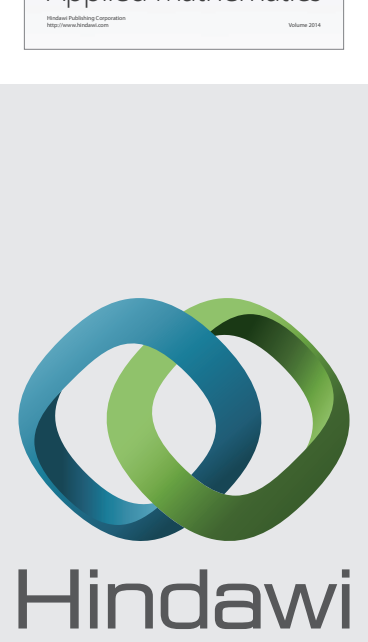

Submit your manuscripts at http://www.hindawi.com
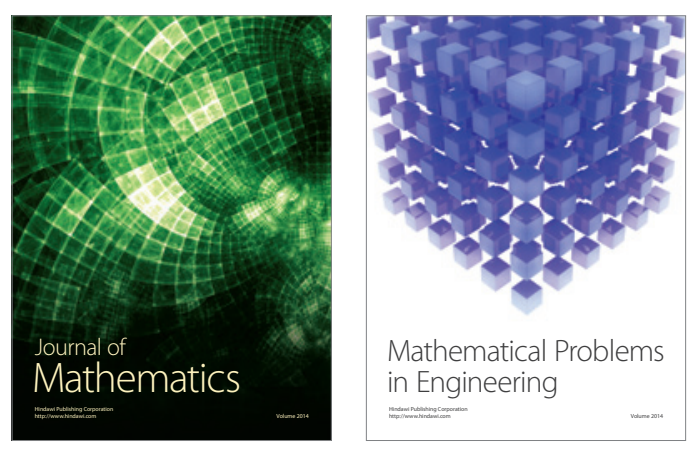

Mathematical Problems in Engineering
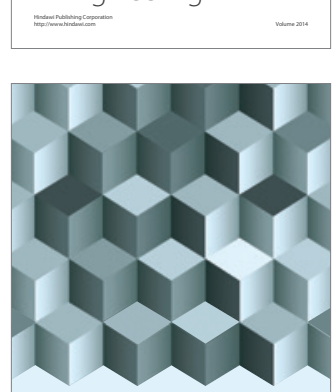

Journal of

Function Spaces
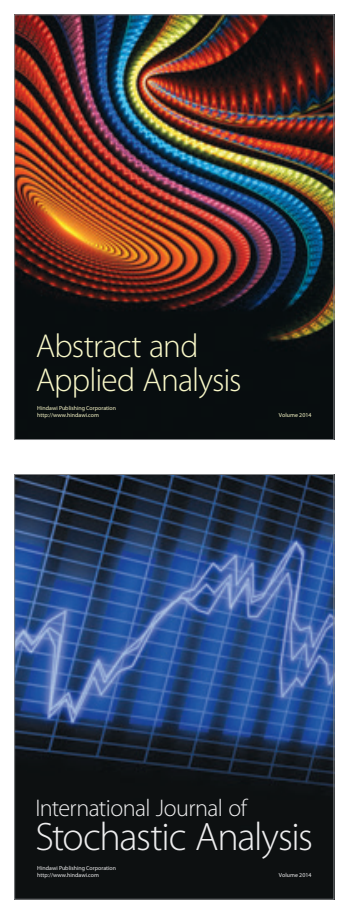

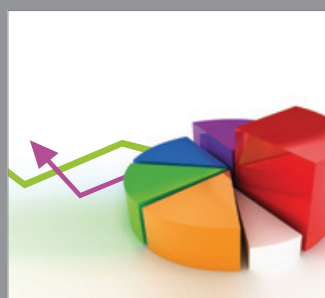

ournal of

Probability and Statistics

Promensencen
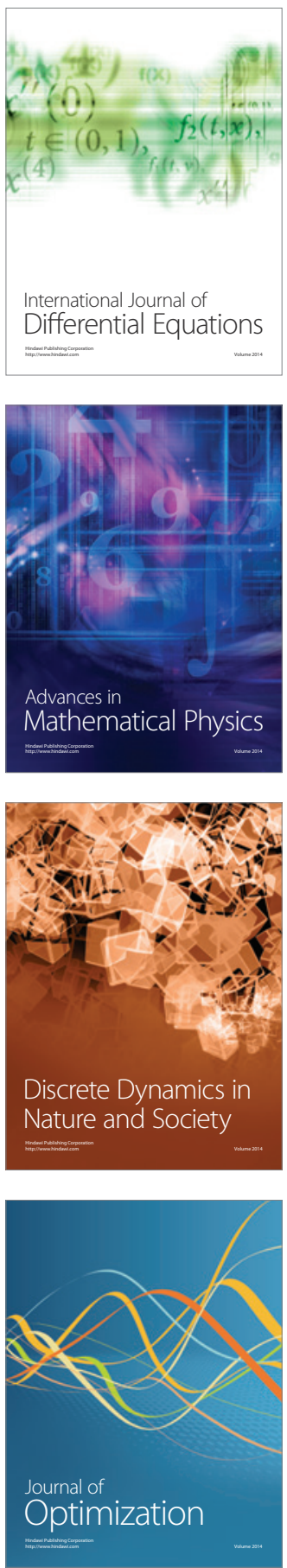